\title{
O trabalho do enfermeiro enquanto docente do ensino superior: uma revisão narrativa
}

\author{
The work of nurses as higher education teachers: a narrative review \\ El trabajo de las enfermeras como profesores de educación superior: una revisión \\ narrativa
}

Caroline Lemos Martins ${ }^{1}$, Maira Buss Thofehrn ${ }^{1}$, Jaqueline Ferreira Ventura Bittencourt ${ }^{2}$, Manuela Gomes Campos Borel2*, Michelle Barboza Jacondino', Zuleyce Maria Lessa Pacheco², Isabel Cristina de Oliveira Arrieira ${ }^{3}$, Diana Cecagno ${ }^{1}$, Thayenne Barrozo Mota Monteiro², Fernanda Ferreira Krepker $^{2}$, Thais Vidal de Oliveira ${ }^{2}$, Amanda Antunes Pereira Madella ${ }^{2}$, Vanessa de Araújo Marques', Adrize Rutz Porto'.

\section{RESUMO}

Objetivo: conhecer o trabalho do enfermeiro que atua no magistério superior. Método: revisão narrativa da literatura que teve como questão norteadora conhecer quais as atividades do enfermeiro docente são abordadas nas publicações científicas no intervalo de 2006-2015. Resultados: à docência em enfermagem em âmbito nacional e internacional é permeada por aspectos como a insuficiência do número de docentes, falta de preparação para atuação nesses espaços, a ênfase na produção científica e as altas demandas decorrentes do trabalho. Nesse sentido, no desenvolvimento das atividades de ensino-pesquisa-extensãoserviço de saúde, os enfermeiros-docentes podem vivenciar sentimentos de estresse, sobrecarga de trabalho e poucos momentos para sua vida pessoal. Conclusão: frente à realidade de trabalho desses profissionais, a partir da literatura, identificou-se que são necessárias reflexões para (re)pensar o papel da docência do enfermeiro.

Palavras-chaves: Enfermagem, Trabalho, Docência em enfermagem, Ensino, Educação.

\begin{abstract}
Objective: to know the work of nurses who work in higher education. Method: narrative review of the literature that had as its guiding question to know which activities of the teaching nurse are addressed in scientific publications in the interval of 2006-2015. Results: nursing teaching at the national and international levels is permeated by aspects such as the insufficient number of teachers, lack of preparation to work in these spaces, the emphasis on scientific production and the high demands arising from work. In this sense, in the development of teaching-research-extension-health service activities, nurse-teachers may experience feelings of stress, work overload and few moments for their personal life. Conclusion: considering the working reality of these professionals, from the literature, it was identified that reflections are necessary to (re) think the role of the teaching of nurses.
\end{abstract}

Keywords: Nursing, Work, Nursing teaching, Teaching, Education.

\section{RESUMEN}

Objetivo: conocer el trabajo de las enfermeras que trabajan en educación superior. Método: revisión narrativa de la literatura que tenía como su pregunta guía para saber qué actividades de la docente de enfermería se abordan en publicaciones científicas en el intervalo de 2006-2015. Resultados: la enseñanza de enfermería a nivel nacional e internacional está impregnada de aspectos como el número insuficiente de docentes, la falta de preparación para trabajar en estos espacios, el énfasis en la producción científica y las altas demandas derivadas del trabajo. En este sentido, en el desarrollo de actividades de servicio de enseñanza-investigación-

1 Universidade Federal de Pelotas (UFPEL), Pelotas - Rio Grande do Sul.

2Universidade Federal de Juiz de Fora (UFJF), Juiz de Fora - Minas Gerais. *E-mail: manu.ufj@@yahoo.com

3Universidade Católica de Pelotas (UCPEL), Pelotas - Rio Grande do Sul.

SUBMETIDO EM: 6/2019

ACEITO EM: 7/2019

PUBLICADO EM: 9/2019

REAS/EJCH | Vol.Sup.32 | e1159 | DOI: https://doi.org/10.25248/reas.e1159.2019 Página 1 de 7 
extensión-salud, las enfermeras-maestras pueden experimentar sentimientos de estrés, sobrecarga de trabajo y algunos momentos de su vida personal. Conclusión: considerando la realidad laboral de estos profesionales, a partir de la literatura, se identificó que las reflexiones son necesarias para (re) pensar el papel de la enseñanza de las enfermeras.

Palabras clave: Enfermería, Trabajo, Docencia en enfermería, Enseñanza, Educación.

\section{INTRODUÇÃO}

A docência em enfermagem é considerada uma prática desafiadora e tem tido destaque nas investigações científicas no Brasil (VIEIRA DRVS e SIQUEIRELLI AS, 2016) e no exterior (SINGH MD, et al., 2014), é uma prática desde o início do século XX, porém ganhou destaque nas últimas duas décadas, devido a necessidade de docentes atualizados e que atendam as reformulações do currículo.

Neste estudo, optou-se por denominar o enfermeiro que desempenha atividades educativas no ensino superior por enfermeiro-docente, o qual concentra a sua prática na realização do ensino de enfermagem aos estudantes. Para isso, espera-se que ele atenda às necessidades de ensino dos mesmos, tenha conhecimento a respeito dos conteúdos estipulados pelas disciplinas e do modelo tradicional de ensino e aprendizagem, para formar enfermeiros que respondam aos requisitos sociais no contexto em que estarão inseridos (DRAGANOV PB e SANNA MC, 2016).

No Brasil, os enfermeiros-docentes de instituições de ensino superior (IES) caracterizam-se por ser uma população feminina, da raça branca, com idade média de 47 anos (entre 26 e 68 anos), casadas, com filhos, possuem Pós-graduação, pertencem à classe de professor adjunto, atuam 40 horas semanais com dedicação exclusiva; estão na instituição há mais de 20 anos e desenvolvem atividades de ensino, pesquisa e extensão (DRAGANOV PB e SANNA MC, 2016). Já a literatura internacional alerta sobre a carência de docentes preparados para atuar na formação de futuros Enfermeiros, como, por exemplo, no Canadá (SINGH MD, et al., 2014).

Almeja-se que o enfermeiro-docente no desempenho de suas atribuições participe de um processo permanente de formação e de reflexão sobre a sua prática profissional. Isso implica em ser crítico-reflexivo sobre as suas ações, práticas didáticas, organizativas e sua relação com o contexto estrutural do ensino superior (LEONELLO VM e OLIVEIRA MAC, 2014).

O processo de docência pode estar envolto por diversos sentimentos, os quais remetem a gostar e possuir amor pela profissão, simpatia, respeito e realização profissional, por outro lado observa-se a falta de reconhecimento profissional, desejo de melhoria salarial, a percepção de desinteresse dos estudantes e a falta de recursos. Esses sentimentos possivelmente tenham relação e sejam desencadeadores de estresse diante das exigências profissionais da docência, do acúmulo de atividades e das múltiplas funções. Desse modo, o ensinar em enfermagem proporciona uma infinidade de desafios, os quais podem suscitar sentimentos de prazer e frustração em decorrência do "peso da incumbência magistral" (ALVES AG, et al., 2016).

Diante disso, o trabalho do enfermeiro-docente tem se mostrado permeado por muitas demandas e múltiplos atores e a partir da literatura, buscou-se trazer reflexões referentes às ações desenvolvidas na docência de enfermagem. Desta forma, o objetivo deste estudo é conhecer o trabalho do enfermeiro que atua no magistério superior.

\section{MÉTODOS}

Trata-se de uma revisão narrativa da literatura, realizada no período de junho a dezembro de 2015, que teve como questão norteadora conhecer quais as atividades do enfermeiro docente são abordadas nas publicações científicas no intervalo de 2006-2015. De acordo com Galvão TF e Pereira MG (2014) esse tipo de revisão permite a investigação de um determinado assunto/problema permitindo novas reflexões e discussões sobre uma temática. Além disso, permite conhecer o estado da arte do objeto de estudo e a sua 
posição histórica, contribuindo para a construção de novas perspectivas sobre o assunto, diferenciando-se da revisão sistemática porquanto não restringe a busca dos estudos a meios muito criteriosos e por utilizar a subjetivação como possibilidade para interpretação dos resultados.

Para o recrutamento dos estudos, fora realizada a busca de artigos nas bases de dados da Literatura Latino-Americana e do Caribe em Ciências da Saúde (LILACS), Biblioteca Nacional de Medicina (NLM) dos Estados Unidos (PubMed), a Biblioteca virtual Scientific Electronic Library Online (SciELO), além de teses e dissertações do Banco de Teses da CAPES. Para isto, foi adotado os seguintes descritores: docentes de enfermagem, educação em enfermagem e educação superior; bem como seus respectivos escritores no idioma inglês: Faculty, Nursing; Education, Nursing; Education, Higher.

Para análise dos dados, inicialmente foi feita uma leitura exploratória e seletiva para verificar o conteúdo dos estudos e selecionar as informações de cada um conforme o objetivo desta pesquisa. Posteriormente, utilizou-se a técnica de análise temática para classificar os resultados, aprofundar o conhecimento e para criação de novas abordagens e conceitos inerentes ao trabalho do enfermeiro que está inserido no ensino superior.

\section{RESULTADOS E DISCUSSÃO}

Com base nisso, foram criadas as seguintes categorias de análise: Contextualização da docência em enfermagem no magistério superior: realidades pelo mundo; e $O$ ser enfermeiro-docente no magistério superior.

\section{Contextualização da docência em enfermagem no magistério superior: realidades pelo mundo}

$\mathrm{Na}$ última década, no contexto norteamericano, evidencia-se nos programas de graduação e pósgraduação dos Estados Unidos da América (EUA) e do Canadá, uma carência de enfermeiros-docentes motivada pela variedade de funções e de responsabilidades, natureza do ambiente acadêmico, insuficiência de recursos financeiros e necessidade de constante qualificação (FANG D e BEDNASH GD, 2014; SINGH MD, et al., 2014).

Outro aspecto identificado para esta carência está no envelhecimento dos profissionais, na incapacidade das instituições em atrair e manter enfermeiros na academia e no aumento da demanda por enfermeiros assistenciais (FANG D e BEDNASH GD, 2014; ROBEZNIEKS A, 2015). E mais, a falta de professores qualificados não permite o aumento do número de matrículas de estudantes de graduação e de pósgraduação, contribuindo para o agravamento da situação, ou seja, para oferecer uma educação de enfermagem de qualidade para a próxima geração de enfermeiros.

Salários baixos e pouco atraentes para profissionais qualificados e a falta de substituição de professores que estão envelhecendo também são aspectos que contribuem para a carência de enfermeiros-docentes, no contexto canadense (SINGH MD, et al., 2014).

Já no Brasil esta realidade é diferenciada, pois a pós-graduação em enfermagem tem quatro décadas e apresenta-se em expansão com aumento do número de programas, egressos e da internacionalização da produção científica. Contudo, ainda há o desafio de ampliar o número de doutores em enfermagem, já que seu crescimento se mostra insuficiente para atender as exigências das IES e das metas estabelecidas pelo Programa Nacional de Pós-graduação. Mas é preciso mencionar que nos cursos de pós-graduação Stricto Sensu, a formação do enfermeiro para a docência no Ensino Superior não tem recebido a devida importância, pois a ênfase tem sido na formação de cientistas ou pesquisadores (MACHADO MH, et al., 2016) que, posteriormente, se tornam 'reféns' de um sistema de produção científica, devido às altas exigências dos órgãos de fomento de pesquisas.

Os docentes de enfermagem lidam com altas demandas decorrentes do seu trabalho, como a expectativa de entender e desenvolver a educação universitária para contornar os problemas sociais vigentes. Espera-se desse profissional uma postura proativa frente ao sistema educativo e, ao mesmo tempo, autônoma e dialógica com vistas à educação de qualidade (MACHADO MH, et al., 2016). 
O ensino torna-se significativo quando suas atividades estão baseadas na integralidade, na correlação entre as atividades da sala de aula, no raciocínio clínico e no pensamento crítico, destacando a importância do papel da formação para a sociedade e para os cuidados de saúde. Essas questões exigem a qualificação dos professores e de um 'novo olhar' sobre a educação de graduação e de pós-graduação, para que os estudantes possam estar preparados para o futuro (ORTEGA MCB, et al., 2015).

Nas últimas décadas, passou-se a exigir que os enfermeiros-docentes nas faculdades e universidades, sejam excelentes professores, ou seja, que se mantenham produtivos, tenham competência clínica e prestem serviços comunitários. Porém, é preciso analisar os prós e os contras do exercício na docência, como benefícios e questões sobre qualidade de vida. Para atender a demanda de atividades, os docentes atuam de 50 a 60 horas por semana desempenhando atividades como supervisão e orientação de estudantes; preparo de conteúdos teóricos e práticos; supervisão e prática clínica; participações em comitês, pesquisa e escrita de artigos científicos; e cuidados à comunidade (ORTEGA MCB, et al., 2015; MACHADO MH, et al., 2016; PADOVANI O e CORREA AK, 2017).

As atividades de ensino-pesquisa-extensão-serviço de saúde foram apresentadas por diversos autores (MACHADO MH, et al., 2016). Ao investigá-las na prática dos enfermeiros-docentes de uma IES brasileira, percebe-se que os professores com mais tempo de atuação na academia relataram o afastamento do ensino teórico, do prático e não desenvolviam atividades de extensão, sendo estas efetivadas por docentes há menos tempo titulados e pouco experientes (MACHADO MH, et al., 2016).

Essas atividades têm seus objetivos dirigidos aos seres humanos, como o desenvolvimento do estudante e o bem-estar dos cidadãos são funcionalmente independentes, mas intrinsecamente relacionadas, sendo necessário que os professores e as instituições de ensino as desenvolvam concomitantemente. Destaca-se que o ensino e a pesquisa são realizados, principalmente, no ambiente acadêmico, enquanto o desempenho da prática clínica da enfermagem tem ocorrido nos hospitais e nos serviços de saúde (MACHADO MH, et al., 2016).

Dessa forma, entende-se que a inspiração para fazer pesquisas deve originar-se da prática clínica e objetivar o avanço da ciência da enfermagem a fim de melhorar os cuidados aos seres humanos. Para promover o avanço da disciplina de enfermagem, é necessário que os enfermeiros-docentes e os enfermeiros assistenciais estabeleçam "parcerias": os benefícios da união entre a academia e a prática clínica são incomensuráveis e podem promover o aperfeiçoamento da disciplina e integrar o ensino à prática da enfermagem (ORTEGA MCB, et al., 2015).

Contudo, para os docentes, a supervalorização da pesquisa em relação ao ensino e a extensão é geradora de sobrecarga, uma vez que a exigência institucional para a realização de pesquisas, nem sempre está aliada a um regime de atividades condizente (LEONELLO VM e OLIVEIRA MAC, 2014).

Os professores consideram que 'ser um pesquisador reconhecido' não garante, necessariamente, um desempenho pedagógico de excelência. Nesse sentido, é preciso colocar a pesquisa a serviço da própria prática, para que possam (re)significá-la, revendo-a, contextualizando-a e confrontando-a com os aspectos condicionantes e determinantes do seu fazer enquanto docentes (LEONELLO VM e OLIVEIRA MAC, 2014).

Em muitos casos, a prioridade dos professores tem sido o ensino e a pesquisa, uma vez que é por meio da pesquisa e da obtenção de financiamento externo que os docentes são recompensados e reconhecidos pelos seus pares. Dessa forma, os enfermeiros-docentes têm priorizado o ensino em programas de pósgraduação em detrimento da graduação (ORTEGA MCB, et al., 2015).

Há o predomínio de atividades de pesquisa e de pós-graduação, além de se submeterem às metas em relação à avaliação dos programas de pós-graduação, à quantidade dos artigos, à competitividade entre pares, ao gerenciamento de projetos financiados e a articulação com pesquisadores de instituições estrangeiras (LEONELLO VM e OLIVEIRA MAC, 2014).

Cabe destacar que as IES públicas no Brasil conferem estabilidade financeira aos enfermeiros-docentes, devido ao plano de carreira, recebimento de melhores salários, quando comparados aos professores de 
instituições privadas, além da carreira ser formalizada e assegurada institucionalmente. Porém, percebe-se claramente uma elevada quantidade de atividades exercidas incompatível com a remuneração (LEONELLO VM e OLIVEIRA MAC, 2014).

Destarte, conforme se observa nos estudos apresentados, os enfermeiros-docentes precisam atender às exigências das IES, dos estudantes e de si próprios para o exercício do seu fazer. Desta forma, importa abordar como os professores estão realizando suas atividades, suas expectativas pessoais e como percebem suas funções no interior das universidades.

\section{O ser enfermeiro-docente no magistério superior}

Para o exercício da docência em enfermagem é preciso que esses indivíduos percebam a sua responsabilidade no ensino aos estudantes; se envolvam com os outros; tenham compromisso com a reforma do ensino; pensem por meio dos problemas; conheçam a si mesmos; sejam um 'símbolo' entre ser o que as pessoas acreditam e ser o que elas precisam; além de agirem conforme seu desejo pessoal e suas emoções. O enfermeiro-docente se auto revela profissionalmente para os discentes e para os usuários por meio da comunicação e de suas atitudes (PADOVANI O e CORREA AK, 2017).

Constata-se que o professor busca reconhecer o seu fazer como algo único e que não pode ser repetido, pois muda a cada dia. É uma profissão em que a coerência e a clareza em relação aos princípios, às crenças e os valores orientam a sua própria vida. Ele necessita ser autêntico e consistente no que diz e no que faz, para poder cumprir os objetivos da formação de recursos humanos na graduação e na pós-graduação e responder às diferentes circunstâncias adversas da vida pessoal e profissional.

Ainda é colocada como responsabilidade do docente, a promoção de um aprendizado significativo aos estudantes, por meio da utilização de estratégias que tornem o processo mais atrativo, como o uso de vídeos, imagens, estudos de caso, artigos, atividades em grupo, estágios em serviços e orientação online. Além disso, acaba sendo exigida clareza na sua comunicação e a capacidade de promover um ambiente em que a aprendizagem é ativa e centrada no estudante. Cabe mencionar que os estudantes de enfermagem almejam encontrar professores com entusiasmo para ensinar, orientar e apoiá-los na sua formação como enfermeiros (MACHADO MH, et al., 2016).

Como o ensino em enfermagem não possui um modelo específico ou um roteiro a seguir, é preciso que os professores experimentem, inovem e busquem melhorias para o futuro, permitindo o avanço do ensino clínico e da profissão. Os enfermeiros-docentes devem ser estimulados a atrair pesquisadores de outras áreas do conhecimento para estabelecerem projetos e programas interdisciplinares, os quais permitirão aumentar sua capacidade para educar e para pesquisar (ORTEGA MCB, et al., 2015).

O objetivo do enfermeiro-docente não é apenas fornecer conhecimentos, mas facilitar aos estudantes e futuros profissionais desenvolver seu próprio conhecimento, por meio de evidências, pesquisas e habilidades (MACHADO MH, et al., 2016).

A enfermagem requer professores qualificados para promover a aprendizagem autônoma de seus estudantes, e ao mesmo tempo, oportunizar que os egressos sejam comprometidos, de tal forma que assumam o papel de protagonistas no desenvolvimento da humanidade e aprendam, desde a sua formação, a participar de um mundo mais humano, equitativo e responsável (GIORDANO DP e FELLI VEA, 2017).

A contribuição do enfermeiro-docente para a formação do estudante e sociedade se encontra em fornecer 'ferramentas intelectuais' e recursos que permitam o desenvolvimento da prática de enfermagem, e para que os futuros enfermeiros critiquem a sua posição sociopolítica (MACHADO MH, et al., 2016). Na realização do ensino em enfermagem, os professores necessitam fazer uma análise crítica das forças sociais, das condições econômicas e do ambiente político que influenciam suas práticas, bem como da sociedade em que vivem e dos cuidados de saúde. A função do enfermeiro-docente não pode ser orientada sem (re)conhecer a essência da enfermagem, isto é, o cuidado. O comprometimento com a prática significa efetivar o que for necessário para o avanço da enfermagem e do ensino, como a pesquisa, o conhecimento, as habilidades e as evidências científicas (ORTEGA MCB, et al., 2015). 
Os professores, ao analisarem o seu fazer, manifestaram aspectos positivos e negativos no exercício da docência. Os positivos estão relacionados à possibilidade de relação entre teoria e prática (práxis), oportunidades de crescimento, satisfação, manifestação da criatividade e habilidades. No tocante aos aspectos negativos, eles perceberam a obrigatoriedade para o sustento, a precarização e a exploração das atividades (GIORDANO DP e FELLI VEA, 2017).

Outro aspecto abordado pelos enfermeiros-docentes refere-se às mudanças e às reformas curriculares nas IES de Enfermagem (CECAGNO D, 2015), as quais são consideradas como fatores geradores de estresse e tensão, pela quantidade de atividades, exigência de qualificação e sobrecarga. Embora as reformas curriculares nas IES tenham exigido dos docentes uma grande quantidade de atividades, favorecendo o esgotamento, os enfermeiros-docentes relatam a percepção de liberdade para atuar nas atividades de ensino, ainda que se sintam frustrados por não terem a oportunidade de avaliar o currículo antes de desenvolvê-lo. A mudança pedagógica dos cursos de enfermagem permite que o estudante seja mais ativo nos métodos de aprendizagem, e o professor necessita se adaptar às mudanças e aos desafios, ininterruptamente.

Como proposta pedagógica, a metodologia ativa participativa e problematizadora é uma ferramenta que auxilia na atuação docente, estimulando a criatividade, a iniciativa e fortalecendo a capacidade didáticopedagógica do professor. É preciso que o enfermeiro-docente tenha a habilidade de considerar a singularidade e as necessidades de cada estudante e acolher a sua unicidade na diversidade de forma a promover um ensino significativo e de acordo com as necessidades dos estudantes (PADOVANI O e CORREA AK, 2017).

A solidariedade e a cooperação entre os colegas foram apontadas por docentes como aspectos que contribuem para a sua qualidade de vida, e que contribuem para a geração de sentimentos de segurança e bem-estar no ambiente de atuação, tornando-o favorável ao desenvolvimento do seu fazer. Nesse sentido, é necessária a promoção de ambientes saudáveis nas IES para o aprimoramento da qualidade de vida do docente, e para que este desenvolva sua práxis diária com sentimentos de satisfação e alegria, favorecendo o processo de ensino e aprendizagem (PADOVANI O e CORREA AK, 2017).

Perceberam-se também alguns conflitos entre os docentes nas IES provenientes da falta de qualificação ou do breve período de tempo no desempenho da docência, sendo esses professores menos favorecidos em relação à remuneração, quantidade de atividades e número de disciplinas do que os professores que atuam há mais tempo. Para os professores titulados, que realizam atividades da docência há mais tempo ou que possuem pesquisas com financiamento, essas se tornam prioridade, a fim de cumprir os requisitos para a progressão na carreira. Isso contribui para a criação de um grupo de professores que têm menos status na instituição, com menor papel na governança do grupo e na contribuição para o funcionamento da universidade. A presença de diferentes grupos de professores, com direitos e privilégios diferentes e cargas de atividades muitas vezes díspares, podem levar a conflitos e tensões dentro da faculdade, contribuindo para a insatisfação dos enfermeiros-docentes (ORTEGA MCB, et al., 2015).

Vale destacar, que como aspectos que melhorariam a qualidade de vida no emprego, são: a obtenção de salário mais elevado, acesso a recursos internos da instituição como bolsas de estudo, redução das atividades no ensino da graduação, estabelecimento de espaços para discussão e estudantes interessados em aprender (GIORDANO DP e FELLI VEA, 2017; PADOVANI O e CORREA AK, 2017).

É necessário que as IES forneçam um ambiente educacional agradável aos docentes, por meio de oportunidades de realização, maior valorização e que os professores avancem na ciência e na prática de enfermagem.

Os programas para o desenvolvimento dos enfermeiros-docentes precisam levar em consideração as políticas institucionais e departamentais, a estrutura organizacional, a cultura acadêmica, o currículo e os conteúdos do ensino e aprendizagem, incluindo os conceitos curriculares, as estratégias básicas de ensino (didática e clínica), o uso de tecnologias, a utilização de simulação e a avaliação do aprendizado dos estudantes (cognitivo, psicomotor e afetivo) (ORTEGA MCB, et al., 2015). 
O processo de reestruturação das IES, guiado por políticas educacionais voltadas à qualidade do ensino, ao invés da mera expansão quantitativa do número de vagas, também impactaria positivamente no fazer docente. Por fim, considera-se importante que os docentes busquem novas estratégias de enfrentamento para os problemas sociais, de saúde e do ensino, podendo identificar e reconhecer mais rápida e eficazmente os problemas e as possíveis soluções, refletindo sobre o seu fazer, (re)significando-o e procurando novas possibilidades de reconstrução de sua prática (LEONELLO VM e OLIVEIRA MAC, 2014).

\section{CONSIDERAÇÕES FINAIS}

Percebe-se, ao analisar a literatura relacionada às atividades dos enfermeiros-docentes no magistério superior que o seu fazer é realizado mediante o contato com outros seres humanos e é influenciado pelas políticas públicas e institucionais, pelos colegas, estudantes e usuários do SUS. Notaram-se muitas demandas e exigências colocadas para esse profissional, em atividades de ensino numa perspectiva do aprendizado significativo, tanto na graduação, como na pós-graduação, pesquisa, extensão e integração ensino-serviço de saúde. Também se percebeu uma supervalorização da pesquisa diante de outras atividades relevantes que fazem parte da docência, além do distanciamento das atividades práticas desse professor, quando se pensa na sua competência associada ao seu papel de enfermeiro nos serviços de saúde. Frente à realidade de trabalho do enfermeiro-docente, a partir da literatura, identificou-se que são necessárias reflexões para (re)pensar o papel da docência, suas atividades e atribuições de uma forma mais humanizada.

\section{REFERÊNCIAS}

1. ALVES AG, et al. O deleite e as agruras de ser professor de enfermagem. Rev Enferm UFPE online. 2016; 10(5):4240-4248. doi: 10.5205/reuol.9284-81146-1-SM.1005sup201610.

2. CECAGNO D. Formação acadêmica do enfermeiro na perspectiva ecossistêmica. [Tese] Rio Grande (Rio Grande do Sul): Universidade Federal do Rio Grande; 2015.

3. DRAGANOV PB, SANNA MC. Competências andragógicas dos docentes enfermeiros que atuam na graduação em enfermagem paulistana. Trab Educ e Saúde. 2016; 14(1):155-182. doi: 10.1590/1981-7746-sip00098.

4. FANG D, BEDNASH GD. Attrition of full-time faculty from schools of nursing with baccalaureate and graduate programs, 2010 to 2011. Nurs Outlook. 2014; 62(3):164-73. doi: 10.1016/j.outlook.2013.12.002.

5. GALVÃO TF, PEREIRA MG. Revisões sistemáticas da literatura: passos para sua elaboração. Epidemiol Serv Saúde. 2014; 23(1):183-184. doi: 10.5123/S1679-49742014000100018.

6. GIORDANO DP, FELLI VEA. Processo de trabalho dos docentes de enfermagem. Rev. Latino-Am. Enfermagem, 2017;25:e2946 DOI: 10.1590/1518-8345.1941.2946.

7. LEONELLO VM, OLIVEIRA MAC. Educação superior em Enfermagem: o processo de trabalho docente em diferentes contextos institucionais. Rev Esc Enferm USP. 2014; 6(48):1093-1102. doi: 10.1590/S0080623420140000700018.

8. MACHADO MH, et al. Aspectos gerais da formação da enfermagem: o perfil da formação dos enfermeiros, técnicos e auxiliares. Enferm. Foco 2016; 6 (2/4): 15-34.

9. ORTEGA MCB, et al. Formação acadêmica do profissional de enfermagem e sua adequação às atividades de trabalho. Rev. Latino-Am. Enfermagem maio-jun. 2015;23(3):404-10 DOI: 10.1590/0104-1169.0432.2569.

10. PADOVANI O, CORREA AK. Currículo E Formação Do Enfermeiro: Desafios Das Universidades Na Atualidade. Sau. \& Transf. Soc., ISSN 2178-7085, Florianópolis, v.8, n.2, mai-ago, p.112-119, 2017.

11. ROBEZNIEKS A. Looming nursing shortage fueled by fewer faculty, training sites. Modern Healthcare. 2015. 45 (4), pp. 9-11.

12. SINGH MD, et al. Empowerment and mentoring in nursing academia. International J Nurs Educ Scholarship. 2014; 1(11):1-11. doi: 10.1515/ijnes-2013-0070.

13. VIEIRA DRVS, SIQUELLI SA. Docência: um desafio humano e político. Rev Intersaberes. 2016; 23(11):408-418. 